\title{
Image Segmentation by Probabilistic Bottom-Up Aggregation and Cue Integration
}

\author{
Sharon Alpert, Meirav Galun, Achi Brandt, and Ronen Basri Member, IEEE
}

\begin{abstract}
We present a bottom-up aggregation approach to image segmentation. Beginning with an image, we execute a sequence of steps in which pixels are gradually merged to produce larger and larger regions. In each step we consider pairs of adjacent regions and provide a probability measure to assess whether or not they should be included in the same segment. Our probabilistic formulation takes into account intensity and texture distributions in a local area around each region. It further incorporates priors based on the geometry of the regions. Finally, posteriors based on intensity and texture cues are combined using "a mixture of experts" formulation. This probabilistic approach is integrated into a graph coarsening scheme providing a complete hierarchical segmentation of the image. The algorithm complexity is linear in the number of the image pixels and it requires almost no user-tuned parameters. In addition, we provide a novel evaluation scheme for image segmentation algorithms attempting to avoid human semantic considerations that are out of scope for segmentation algorithms. Using this novel evaluation scheme we test our method and provide a comparison to several existing segmentation algorithms.
\end{abstract}

Index Terms—computer vision, image segmentation, cue integration, segmentation evaluation.

\section{INTRODUCTION}

Image segmentation algorithms aim at partitioning an image into regions of coherent properties as a means for separating objects from their backgrounds. As objects may be separable by any of a variety of cues, be it intensity, color, texture, or boundary continuity, many segmentation algorithms were developed and a variety of techniques were utilized including: clustering [1], [2], Markov random fields [3], [4], variational methods [5], [6] and level set methods [7].

A different approach that has recently gained popularity is to apply graph algorithms to segmentation. Typically, given an image a graph is constructed in which a node represents a pixel and weighted edge is used to encode an "affinity" measure between nearby pixels. The image is then segmented by minimizing a cost associated with partitioning the graph into

- S. Alpert, M. Galun, A. Brandt, and R. Basri are with the Department of Computer Science and Applied Mathematics, The Weizmann Institute of Science, P.O. Box 26, Rehovot 76100, Israel. E-mail: sharon.alpert, meirav.galun, achi.brandt, ronen.basri@weizmann.ac.il.

- A. Brandt is also with the Department of Mathematics, University of California, Los Angeles, 520 Portola Plaza, Los Angeles, California, U.S.A. subgraphs. In the simpler version, the cost is the sum of the affinities across the cut [8], [9]. Other methods normalize the cut cost either by dividing it by the overall area or the boundary length of the segments [10], [11] or by normalizing the cut cost with measures derived from the affinities within the segments [8], [12], [13], [14]. A prominent example for the latter approach, is the normalized-cut algorithm [15], but attaining a globally optimal solution for the normalized-cut measure and similar measures is known to be NP-hard even for planar graphs. While polynomial time approximations to the normalizedcuts are available, the computational requirement remains high. In attempt to overcome this [16] uses connections at different scales to decrease the complexity of the graph. Another multiscale approach, presented in [17], utilizes a fast multilevel solver based on Algebraic Multigrid (AMG) to construct a hierarchy of segmentations, where average image cues are estimated with minimal mixing of segments statistics. The average cues then influence the construction of further scales in the hierarchy to produce a more reliable segmentation.

Methods like [15], [17] have been designed to 
utilize and combine multiple cues. Typically in such algorithms, each cue is handled by a separate module whose job is to assess the coherence of nearby pixels or regions according to that cue, and a segmentation decision is obtained by incorporating these similarities into a combined measure. Careful design of these modules along with the use of appropriate optimization methods has led to notable successes, but the challenge of reliably segmenting objects in a variety of natural images still lies ahead.

The utilization of multiple cues aggravates an old problem. In many multi-cue segmentation algorithms each module comes with its own set of parameters, and those join an additional set of parameters intended to control the relative influence of each module. These parameters may depend non-trivially on the particular statistics of the input image, or even the statistics of different regions in the same image. While existing methods may be robust to changes in some of those parameters, segmentation results in many cases may depend critically on the proper assignment of parameter values. The common practice is to leave those parameters to be set by the user, but in effect most users leave the parameters in their default values. Allowing these parameters to automatically adapt to an image (or even locally to image portions) can greatly simplify the use of segmentation algorithms and potentially allow them to consistently provide better results. Indeed, recent algorithms attempt to achieve parameter-free segmentation, either by adapting to a specific class of images (e.g., [18]) or, in the case of natural images, by relying on a training set that includes a variety of manually segmented images (e.g., [19]). A different stream of work uses cluster analysis to estimating a global set of parameters (e.g., stability criteria in [20]).

In this paper we explore a different approach which relies primarily on local information available within the image to be segmented. We present a probabilistic approach to segmentation that is almost parameter free. Beginning with an image, we execute a sequence of steps in which pixels are gradually merged to produce larger and larger regions. In each step we consider pairs of adjacent regions and provide a probability measure to assess whether or not they should be included in the same segment. We illustrate this method by constructing modules to handle intensity
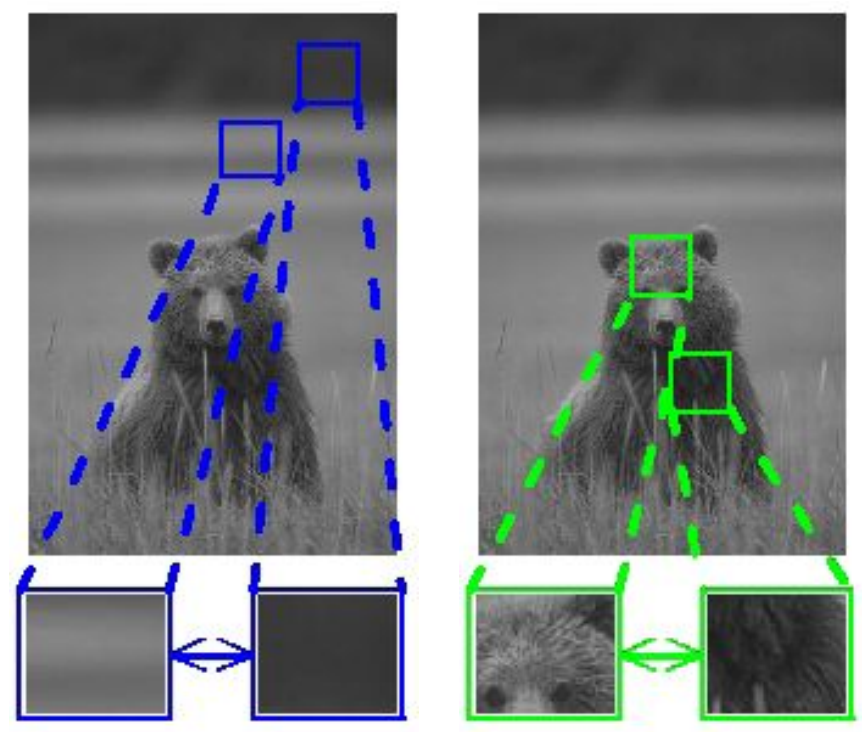

Fig. 1: The importance of adaptive, local cue integration. Left: two patches that can be distinguished by intensity (the patches have uniform textures). Right: two patches with similar texture that should be merged despite their different intensities (due to lighting).

contrast and texture differences, and use an adaptively controlled "mixture of experts"-like approach to integrate the different cues and reach unified segmentation decisions. To illustrate the importance of adaptive, local cue integration consider the example in Figure 1, which shows two pairs of regions. The left pair can be distinguished by intensity cues, whereas the right pair of patches, which have similar texture, should be merged despite their different intensities.

Our approach is designed to work with bottom-up merge strategies for segmentation. A large number of methods approach segmentation using bottom-up merge strategies, beginning with the classic agglomerative clustering algorithm [21] to watershed [22], [23] and region growing (including methods that use probabilistic approaches [24], [25] to more recent algebraic multigrid inspired aggregation [17]. Merge algorithms generate a hierarchy of segments, allowing subsequent algorithms to choose between possible segmentation hypotheses. For implementation we adapt the coarsening strategy introduced in [17], as it enables incorporating at every level of the hierarchy 
measurements appropriate to the scale at that level.

Another contribution of our paper is a novel segmentation evaluation scheme that is suited for the evaluation of data-driven segmentation algorithms. Evaluating the results produced by segmentation algorithms is challenging, as it is difficult to come up with canonical test sets providing ground truth segmentations. This is partly because manual delineation of segments in everyday complex images can be laborious and often tend to incorporate semantic considerations which are beyond the scope of data driven segmentation algorithms. In this paper we propose an evaluation scheme that is based on an image dataset which was specifically chosen, such that the human annotations would avoid semantic considerations. We test our parameter-free approach on this database and compare our results to several existing algorithms. This paper is based on [26], where in this paper we have expanded the evaluation test set. This results in a comprehensive segmentation evaluations scheme that accounts for changes in both scale and low-level cues.

\section{Probabilistic framework}

We consider a bottom-up aggregation approach to image segmentation. In this approach beginning with an image, we execute a sequence of steps in which pixels are gradually merged to produce larger and larger regions. In this section we focus on one step of such a procedure, in which a division of the image into a set of regions $\mathcal{R}=\left\{R_{1}, R_{2}, \ldots, R_{n}\right\}$ is given, along with a set of observations, $\overrightarrow{\mathcal{H}}_{i} \in \mathbb{R}^{d}$ for each region $R_{i}(i=1 \ldots n)$. Our objective is to further merge these regions to produce larger regions of coherent properties.

To achieve this goal we consider pairs of adjacent regions, $R_{i}$ and $R_{j}$, and provide a measure to assess whether or not they should be merged into a single segment. We define a binary random variable $\mathbf{s}_{i j}$ that assumes the values $\mathbf{s}_{i j}^{+}$if $R_{i}$ and $R_{j}$ belong to the same segment and $\mathbf{s}_{i j}^{-}$if they do not. We then wish to estimate the probability $P\left(\mathbf{s}_{i j}^{+} \mid \overrightarrow{\mathcal{H}}_{i}, \overrightarrow{\mathcal{H}}_{j}\right)$ which we will use to determine whether or not to merge the two regions based on their respective properties.

Since segmentation decisions may be affected by several cues, we need a method to integrate the different cues. Here we consider both intensity and texture cues and integrate them using the "mixture of experts"-like model, as follows.

$$
\begin{aligned}
& P\left(\mathbf{s}_{i j}^{+} \mid \overrightarrow{\mathcal{H}}_{i}, \overrightarrow{\mathcal{H}}_{j}\right)= \\
& \quad \sum_{k} P\left(\mathbf{s}_{i j}^{+}, c_{k} \mid \overrightarrow{\mathcal{H}}_{i}, \overrightarrow{\mathcal{H}}_{j}\right)= \\
& \sum_{k} P\left(\mathbf{s}_{i j}^{+} \mid \overrightarrow{\mathcal{H}}_{i}, \overrightarrow{\mathcal{H}}_{j}, c_{k}\right) P\left(c_{k} \mid \overrightarrow{\mathcal{H}}_{i}, \overrightarrow{\mathcal{H}}_{j}\right) .
\end{aligned}
$$

This equation implies that the probability of a merge is determined separately for each cue $c_{k}$, and the term $P\left(c_{k} \mid \overrightarrow{\mathcal{H}}_{i}, \overrightarrow{\mathcal{H}}_{j}\right)$ enables us to adjust the influence of each cue dynamically according to the characteristics of the regions.

To evaluate the probability of a merge for each cue we apply Bayes' formula:

$$
P\left(\mathbf{s}_{i j}^{+} \mid \overrightarrow{\mathcal{H}}_{i}, \overrightarrow{\mathcal{H}}_{j}, c_{k}\right)=\frac{L_{i j}^{+} P\left(\mathbf{s}_{i j}^{+} \mid c_{k}\right)}{L_{i j}^{+} P\left(\mathbf{s}_{i j}^{+} \mid c_{k}\right)+L_{i j}^{-} P\left(\mathbf{s}_{i j}^{-} \mid c_{k}\right)}
$$

where $L_{i j}^{ \pm} \triangleq p\left(\overrightarrow{\mathcal{H}}_{i}, \overrightarrow{\mathcal{H}}_{j} \mid \mathbf{s}_{i j}^{ \pm}, c_{k}\right)$ denote the likelihood densities given $\mathbf{s}_{i j}^{ \pm}$respectively. These likelihoods are determined locally according to properties of surrounding regions. We further use a prior that is independent of cue, $P\left(\mathbf{s}_{i j} \mid c_{k}\right)=P\left(\mathbf{s}_{i j}\right)$, and determine this prior based on the geometry of the two regions, i.e., their relative length of common boundaries.

In the remainder of this section we elaborate on how we model the likelihood densities, the cue arbitration, and prior probabilities.

\section{1 likelihood densities}

Below we describe how we derive the likelihood densities for each of our cues, intensity and texture. Both likelihoods are determined from the image by local properties of surrounding regions. Roughly speaking, the underlying principle in our choice of likelihoods is that in principle we consider it likely that a region would merge with its most similar neighbor, while we consider it unlikely that a region would merge with all of its neighbors. We further define these likelihoods to be symmetric and take scale considerations into account.

\subsubsection{Intensity likelihood density}

For two neighboring regions $R_{i}$ and $R_{j}$, denote their average intensities by $\overline{I_{i}} \in \overrightarrow{\mathcal{H}}_{i}$ and $\overline{I_{j}} \in \overrightarrow{\mathcal{H}}_{j}$, we model 
both likelihoods $L_{i j}^{ \pm}$for the case of intensity in (2) as zero mean Gaussian density functions of their average intensity difference $\Delta_{i j}=\overline{I_{i}}-\overline{I_{j}}$, i.e.,

$$
L_{i j}^{ \pm}=p\left(\Delta_{i j} \mid \mathbf{s}_{i j}^{ \pm}\right)=\mathcal{N}\left(0, \sigma_{i j}^{ \pm}\right),
$$

where the standard deviations $\sigma_{i j}^{ \pm}$are given as sums of two terms:

$$
\sigma_{i j}^{ \pm}=\sigma_{\text {local }}^{ \pm}+\sigma_{\text {scale }} .
$$

To determine $\sigma_{\text {local }}^{+}$we consider for region $i$ its neighbor whose average intensity is most similar (and likewise for region $j$ ). Denote the minimal external difference by $\Delta_{i}^{+}=\min _{k}\left|\Delta_{i k}\right|$, where $k$ denotes the immediate neighbors of $i$, then

$$
\sigma_{\text {local }}^{+}=\min \left(\Delta_{i}^{+}, \Delta_{j}^{+}\right) .
$$

To determine $\sigma_{\text {local }}^{-}$, we take into account for region $i$, and similarly for region $j$, the average intensity difference over all of its neighbors, $\Delta_{i}^{-}$, i.e.,

$$
\Delta_{i}^{-}=\frac{\sum_{k} \tau_{i k} \Delta_{i k}}{\sum_{k} \tau_{i k}},
$$

where $\tau_{i k}$ denotes the length of the common boundaries between $R_{i}$ and each of its neighbors $R_{k}$ (see Section 3.2). Then we define

$$
\sigma_{\text {local }}^{-}=\frac{\Delta_{i}^{-}+\Delta_{j}^{-}}{2} \text {. }
$$

We further increase the standard deviation of each of the likelihoods by $\sigma_{\text {scale }}$. Suppose the image contains additive zero mean Gaussian noise with known standard deviation $\sigma_{n o i s e}$. As we consider larger regions the effect of the noise on the average intensity of the regions shrinks. In particular, for a region $R_{i}$ containing $\Omega_{i}$ pixels the standard deviation of the noise added to the average intensity is approximately

$$
\sigma_{\text {noise }}^{R_{i}}=\frac{\sigma_{\text {noise }}}{\sqrt{\Omega_{i}}} .
$$

Hence we choose

$$
\sigma_{\text {scale }}=\frac{\sigma_{\text {noise }}}{\min \left(\sqrt{\Omega_{i}}, \sqrt{\Omega_{j}}\right)} .
$$

$\sigma_{\text {noise }}$ can be estimated in a number of ways ([27]), e.g., by taking the minimal standard deviation across random image patches. Throughout our experiments, however, we used a constant value.

\subsubsection{Texture likelihood densities}

To account for texture we apply to each region $R_{i}$ a bank of edge filters and store their total absolute responses in a histogram $\mathbf{h}_{i} \in \overrightarrow{\mathcal{H}}_{i}$ containing $\nu=|\mathbf{h}|$ bins (the filters we use are specified in Section 3.2). To measure the difference between two histograms $\mathbf{h}_{i}$ and $\mathbf{h}_{j}$ we use a measure similar to the $\chi^{2}$ difference test [28]:

$$
D_{i j}=\sum_{k}\left(\frac{\mathbf{h}_{i}(k)-\mathbf{h}_{j}(k)}{\mathbf{h}_{i}(k)+\mathbf{h}_{j}(k)}\right)^{2} .
$$

Assuming that each response is distributed normally $\mathbf{h}_{i}(k) \sim \mathcal{N}\left(\mu_{k}, \sigma_{k}\right)$ we construct two new $\chi_{\nu}^{2}$ variables ( $\nu$ denotes the number of degrees of freedom), which are expressed as products of the form $\alpha^{+} D_{i j}$ and $\alpha^{-} D_{i j}$ as follows. We use again the concept that two regions with similar texture are more likely to be in the same segment. Recall, that the $\chi_{\nu}^{2}$ distribution receives its maximum at $\nu-2$. Let $D_{i}^{+}=\min _{k} D_{i k}$ we model $L_{i j}$ in (2) by

$$
L_{i j}^{ \pm}=p\left(D_{i j} \mid \mathbf{s}_{i j}^{ \pm}\right)=\chi^{2}\left(D_{i j} \alpha^{ \pm}\right),
$$

where $\alpha^{+}=\frac{\nu-2}{\min \left(D_{i}^{+}, D_{j}^{+}\right)}$guaranties that the closest region in terms of texture will receive the highest likelihood. Similarly, we set $\alpha^{-}$to reflect the difference in texture relative to the entire neighborhood. We therefore compute the average texture difference in the neighborhood, weighted by the length of the common boundaries between the regions

$$
D_{i}^{-}=\frac{\sum_{k} \tau_{i k} D_{i k}}{\sum_{k} \tau_{i k}}
$$

and set $\alpha^{-}=\frac{\nu-2}{\frac{1}{2}\left(D_{i}^{-}+D_{j}^{-}\right)}$.

\subsection{Prior}

We determine the prior $P\left(\mathbf{s}_{i j}^{ \pm}\right)$according to the geometry of the regions. Roughly speaking, a-priori we consider neighboring regions with long common boundaries more likely to belong to the same segment than regions with short common boundaries. Recall that $\tau_{i j}$ denotes the length of the common boundary of $R_{i}$ and $R_{j}$. Hence, we define the prior as:

$$
P\left(\mathbf{s}_{i j}^{+}\right)=\frac{\tau_{i j}}{\min \left(\sum_{k} \tau_{i k}, \sum_{k} \tau_{j k}\right)} .
$$




\subsection{Cue integration}

As we mentioned in the beginning of Section 2 we integrate segmentation decisions from different cues using a local "mixture of experts"-like model. This model allows us to control the influence of each cue and adapt it to the information contained in each region. Thus, for example, when we compare two textured regions we can discount the effect of intensity and by this overcome brightness variations due to lighting.

To determine the relative influence of every cue we need to estimate $P\left(c_{k} \mid \overrightarrow{\mathcal{H}}_{i}, \overrightarrow{\mathcal{H}}_{j}\right)$. To that end we want to evaluate for each region whether or not it is characterized by texture. For each region $R_{i}$ we calculate a 256-bin histogram of local gradients magnitudes $G^{i}$ inside the region. Since, textured regions are often characterized by significant edge responses in different orientations and scales [29], we expect the gradients magnitude histogram of a non-textured region to be fairly sparse. To measure sparseness we first normalize the histogram $\left(\sum_{k} G_{k}^{i}=1\right)$ and apply to each region the measure [30]:

$$
S_{i}=\frac{1}{\sqrt{n}-1}\left(\sqrt{n}-\frac{\left\|G^{i}\right\|_{1}}{\left\|G^{i}\right\|_{2}}\right),
$$

where $n$ denotes the number of bins in $G^{i}$ and $\left\|G^{i}\right\|_{p}$ denotes the $\ell_{p}$ norm of $G^{i}$. Note that we exclude from this calculation pixels which lie along the boundary of a region since they may reflect boundary gradients rather than texture gradients. Finally, we combine these measures by

$$
p\left(c_{1} \mid \overrightarrow{\mathcal{H}}_{i}, \overrightarrow{\mathcal{H}}_{j}\right)=\min \left(P\left(c_{1} \mid \overrightarrow{\mathcal{H}}_{i}\right), P\left(c_{1} \mid \overrightarrow{\mathcal{H}}_{j}\right)\right),
$$

with $c_{1}$ denotes the intensity cue. We further model the individual probabilities using the logistic function:

$$
p\left(c_{1} \mid \overrightarrow{\mathcal{H}}_{i}\right)=\frac{1}{\left(1-e^{-\left(a S_{i}+b\right)}\right)} .
$$

To estimate the constant parameters $a, b$ we used 950 random patches from the Brodatz data set [31] and a similar number of non-textured patches selected manually from random images as a training set. A sample from this set is shown in Figure 2. Then, a maximum likelihood estimation (MLE) regression was used to estimate $a$ and $b$. The values we estimated are $a=41.9162$ and $b=-37.1885$, these parameters were
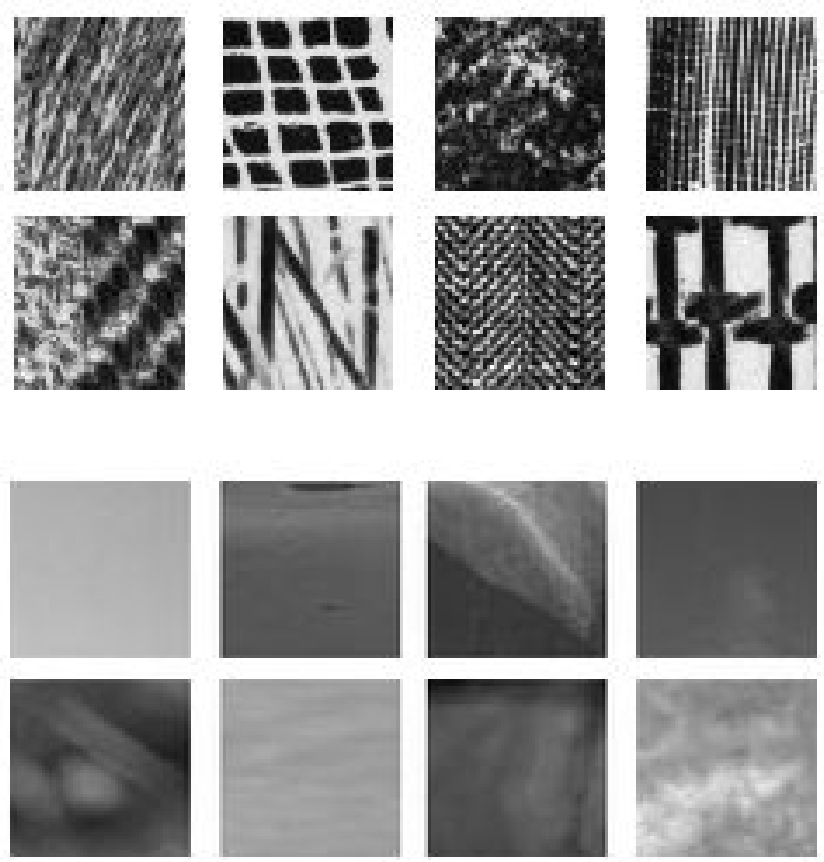

Fig. 2: Samples from the training set used to determine the logistic function (16). Top: texture samples. Bottom: intensity samples

used throughout our experiments.

\section{Algorithm}

Our probabilistic framework is designed to work with any merge algorithm for segmentation. Here we use the merge strategy suggested for the Segmentation by Weighted Aggregation (SWA) algorithm [17], [32], which employs a hierarchy construction procedure inspired by Algebraic Multigrid (AMG) solutions for differential equations [33]. The SWA algorithm begins with a weighted graph representing image pixels, and in a sequence of steps creates a hierarchy of smaller ("coarse") graphs with soft relations between nodes at subsequent levels. The edge weights in the new graphs are determined by inheritance from previous levels and are modified based on regional properties. These properties are computed recursively as the merge process proceeds. Below we use the coarsening strategy of the SWA algorithm and modify it to incorporate our probabilistic framework. In particular, we 
use as edge weights the posterior probabilities defined in Section 2. We produce the coarser graphs using the coarsening strategy of SWA, but replace inheritance of weights by computing new posteriors. Overall, we achieve a method that is as efficient as the SWA algorithm, but relies on different, probabilistic measures to determine segmentation and requires almost no user tuned parameters.

\subsection{Graph coarsening}

Given an image we begin by constructing a 4connected graph $G^{[0]}=\left(V^{[0]}, E^{[0]}\right)$, in which every pixel is represented by a node and neighboring pixels are connected by an edge. Using the formulation described in Section 2, we associate a weight $p_{i j}$ with each edge $e_{i j}$,

$$
p_{i j}=P\left(\mathbf{s}_{i j}^{+} \mid \overrightarrow{\mathcal{H}}_{i}, \overrightarrow{\mathcal{H}}_{j}\right),
$$

utilizing a uniform prior at this first stage.

We then execute repeatedly the following steps in order to progressively construct smaller graphs, $G^{[1]}, G^{[2]}, \ldots$, each contains about half the number of nodes in the preceding graph, along with interpolation weights relating the elements in each two consecutive graphs

Coarse node selection: Given a graph $G^{[s-1]}=$ $\left(V^{[s-1]}, E^{[s-1]}\right)$ we begin the construction of $G^{[s]}$ by selecting a set of seed nodes $C \subset V^{[s-1]}$, which will constitute the subsequent level. Let us denote the unselected nodes by $F=V^{[s-1]}-C$. Then, the selection of the seeds is guided by the principle that each $F$-node should be "strongly coupled" to nodes in $C$, i.e., for each node $i \in F$ we require that

$$
\frac{\sum_{j \in C} p_{i j}}{\sum_{j \in V^{[s-1]}} p_{i j}}>\psi
$$

where $\psi$ is a parameter (usually, $\psi=0.2$ ). The construction of $C$ is done using a sequential scan of the nodes in $V^{[s-1]}$, adding to $C$ every node that does not satisfy (18) with respect to the nodes already in $C$. The scanning order may be determined according to a certain desired property of the regions, e.g., by decreasing size of the nodes, influencing $C$ to contain larger regions.

Once $C$ is selected we construct $V^{[s]}$ to include copies of the nodes in $C$. To simplify notations we assume without loss of generality that the nodes $1,2, \ldots,|C| \in V^{[s-1]}$ compose $C$, while the rest are in $F$. This allows us to assign the same index to nodes in $V^{[s]}$.

Inter-level interpolation: We determine the inter-level interpolation weights as follows. For each node $i \in F$ we denote by $N_{i}=\left\{j \in C \mid p_{i j}>0\right\}$ its "coarse neighborhood." We define a matrix $T^{[s-1][s]}$ of size $\left|V^{[s-1]}\right| \times|C|$ by:

$$
t_{i j}= \begin{cases}p_{i j} / \sum_{k \in N_{i}} p_{i k} & \text { for } i \in F, j \in N_{i} \\ 1 & \text { for } i \in C, j=i \\ 0 & \text { otherwise. }\end{cases}
$$

Computing regional properties: For each coarse node $i \in V^{[s]}$ we compute intensity and texture properties by averaging over the properties of its descendants. These are stored in a feature vector $\overrightarrow{\mathcal{H}}_{i}{ }^{[s]}$. We further elaborate on the computation of regional properties in Section 3.2.

Coarse graph probabilities: Finally, the edge weights of the coarse graph are determined. Unlike the SWA, we do not inherit those weights from the previous level. Instead we compute new posteriors for the nodes of the coarse graph. For every pair of neighboring nodes, $i, j \in V^{[s]}$ we assign the weight

$$
p_{i j}^{[s]}=P\left(\mathbf{s}_{i j}^{+} \mid \overrightarrow{\mathcal{H}}_{i}^{[s]}, \overrightarrow{\mathcal{H}}_{j}^{[s]}\right) .
$$

These posteriors are determined, as is described in Section 2, using the newly computed regional properties.

\subsection{Features}

In order to determine the edge weights at every level we need to compute posterior probabilities as in Section 2. The computation of these posteriors uses the average intensity and histogram of filter responses computed for every region, as well as the length of boundaries between every two neighboring regions. The merge strategy described above enables us to compute these properties efficiently for every node, by averaging the same properties computed for its descendants. The properties we are using can be divided into two kinds: unary features, computed for a single region, e.g., the average intensity or histogram of filter responses, and binary features, e.g., the length of the common boundary between two regions. Below 
we describe how we compute these properties during the coarsening process.

\subsubsection{Unary features}

Our intensity and texture features can be obtained by summation of the corresponding feature values over all pixels in a region. For every node $k$ at scale $s$ we can compute such a feature by taking a weighted sum of the feature values of its descendants. Specifically, for a pixel $i$ we denote its feature value by $q_{i}$. Denote by $T_{i k}^{[s]}$ the extent to which pixel $i$ belongs to the region $k$ at scale $s, T_{i k}^{[s]}$ can be determined from the matrix product $T^{[s]}=\prod_{m=0}^{s-1} T^{[m][m+1]}$. We further denote by $\bar{Q}^{[s]}$ the weighted average of $q_{i}$ for all pixels $i$ which belong to region $k$, i.e.,

$$
\bar{Q}_{k}^{[s]}=\frac{\sum_{i} t_{i k}^{[s]} q_{i}}{\sum_{i} t_{i k}^{[s]}} .
$$

Then, $\bar{Q}_{k}^{[s]}$ can be computed using the following recursive formula:

$$
\bar{Q}_{k}^{[s]}=\frac{\sum_{j} t_{j k} \Omega_{j}^{[s-1]} \bar{Q}_{j}^{[s-1]}}{\sum_{j} t_{j k} \Omega_{j}^{[s-1]}},
$$

where $\Omega_{j}^{[s-1]}$ denotes the size of aggregate $j$ at scale $s-1$, which is computed recursively in a similar way, and $t_{j k}$ is the element $j k$ in the matrix $T^{[s-1][s]}$.

We use this recursive formulation to compute the following features:

Average intensity: Starting with the intensity value $I_{i}$ at each pixel $i$ at scale 0 , the quantity $\bar{I}_{k}^{[s]}$ provides the average intensity in a region $k$ at scale $s$.

Texture: For each pixel, we measure short Sobel-like filter responses, following [32], in four orientations $0, \frac{\pi}{2}, \frac{\pi}{4}, \frac{3 \pi}{4}$ and accumulate them recursively to obtain a 4-bin histogram for each region at each scale. Since filter responses at points near the boundaries of a segment may respond strongly to the boundaries, rather than to the texture at the region we employ a topdown cleaning process to eliminate these responses from the histogram.

\subsubsection{Binary features}

To determine the prior probability $P\left(\mathbf{s}_{i j}^{ \pm}\right)$we need to compute for every pair of neighboring regions the length of their common boundaries. Beginning at the level of pixels, we initialize the common boundaries $\tau_{i j}$ of two neighboring pixels to 1 (we use 4-connected pixels) and 0 otherwise. Then, for every neighboring regions $k$ and $l$ at scale $s$ we compute the length of their common boundaries using the formula:

$$
\tau_{k, l}^{[s]}=\sum_{i j} \tau_{i j}^{[s-2]},
$$

where the indices $i$ and $j$ sum respectively over all the maximal decedents of $k$ and $l$ of level $s-2$; i.e. $i$ and $j$ are aggregates of level $s-2$ that respectively belong to $k$ and $l$ with largest interpolation weights relative to all other nodes of scale $s$. Again, this property can be accumulated recursively from one level to the next.

\section{EXPERIMENTS}

\subsection{Segmentation benchmark}

Evaluating the results produced by segmentation algorithms is challenging, as it is difficult to come up with canonical test sets providing ground truth segmentations. This is partly because manual delineation of segments in everyday complex images can be laborious. Furthermore, people often tend to incorporate into their segmentations semantic considerations which are beyond the scope of data-driven segmentation algorithms. For this reason many existing algorithms show only few segmentation results. An important attempt to produce an extensive evaluation database for segmentation was recently done at Berkeley [34]. This database however has its own limitations, as can be noticed by the differences between subjects. In many cases images are under-segmented, and semantic considerations seem to dominate the annotated segmentations (see Fig. 3). Another benchmark was presented in [35]. In this benchmark segmentation is evaluated by judging the accuracy of segmenting only the most salient object in each image (determined by two human subjects), although the images in this benchmark often contain several perceptually salient objects. In addition, the issue of fragmentation is not considered in the evaluation methodology.

To evaluate our method and compare it to recent algorithms we have compiled a database containing 200 gray level images along with ground truth segmentations. The database was designed to contain a 


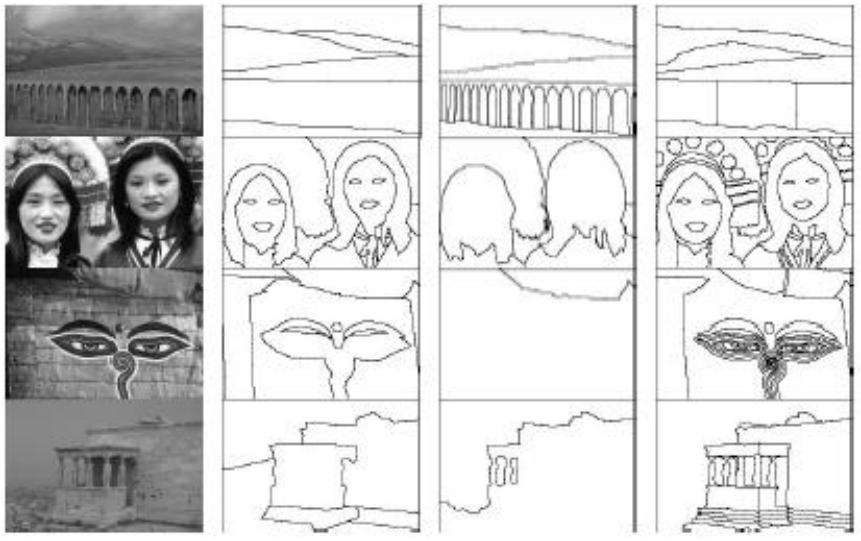

Fig. 3: Example of human annotations from the Berkeley segmentation dataset (on the left are the original images). Note the large variations in human annotations and the difference in the underline number of segments.

variety of images with objects that differ from their surroundings by either intensity, texture, or other low level cues. To avoid potential ambiguities we only selected 100 images that clearly depict one object in the foreground and another 100 images that clearly depict two foreground objects, often with noticeable scale differences.

To obtain ground truth segmentation we asked about 100 subjects to manually segment the images into either two or three classes, depending on the image, with each image segmented by three different human subjects. We further declared a pixel as foreground if it was marked as foreground by at least two subjects. A sample from the database is shown in Figure 4. The complete database and the human segmentation is available at [36].

We evaluated segmentation results by assessing their consistency with the ground truth segmentation and by their amount of fragmentation. For consistency we used the F-measure [37]. Denote by $P$ and $R$ the precision and recall values of a particular segmentation than the F-measure is defined as

$$
F=\frac{2 R P}{P+R} .
$$

The amount of fragmentation is given simply by the number of segments needed to cover a single foreground object.

\subsection{Evaluation}

We applied our segmentation algorithm to all 200 images in the database and compared our results to several state of the art algorithms including:

1) Segmentation by weighted aggregation (SWA)[17]. We tested two variants, one which uses the full range of features described in [32] (denoted by SWA V1) and a second variant which relies only on features similar to the ones used by our method, i.e., intensity contrast and filter responses (denoted by SWA V2) (WINDOWS implementation at www.cs.weizmann.ac.il/ vision/SWA/).

2) Normalized cuts segmentation including intervening Contours [28] (Matlab implementation at www.cis.upenn.edu/ jshi/).

3) Mean-Shift [38]. This method uses intensity cues only (EDISON implementation at www.caip.rutgers.edu).

4) Contour Detection and Hierarchical Image Segmentation (Gpb) [39] (Matlab implementation at www.cs.berkeley.edu/ arbelaez/UCM.html)

For our method only a single parameter, $\sigma_{\text {noise }}$ needed to be specified. We set this parameter to a fixed value for all images $\left(\sigma_{\text {noise }}=18\right)$. The other algorithms were run with several sets of parameters. The normalized cuts algorithm was run with the a range of parameters around the expected number of classes. For the MeanShift and SWA we tested roughly 40 different sets of parameters. In each case we selected for the final score for each dataset the set of parameters that gave the best performance for that dataset (i.e. one or two objects).

We performed two tests. In the first test we selected in each run the segment that fits the foreground the best, according to the F-measure score. Note that for the two objects dataset, we selected the best segment separately for each foreground object. The results of the single segment coverage test for the both datasets are given in Tables 1-2.

In this test our method achieved the highest averaged F-measure score on the single object dataset and came in second on the two object dataset. The $\mathrm{Gpb}$ algorithm, which achieved the best score in the 

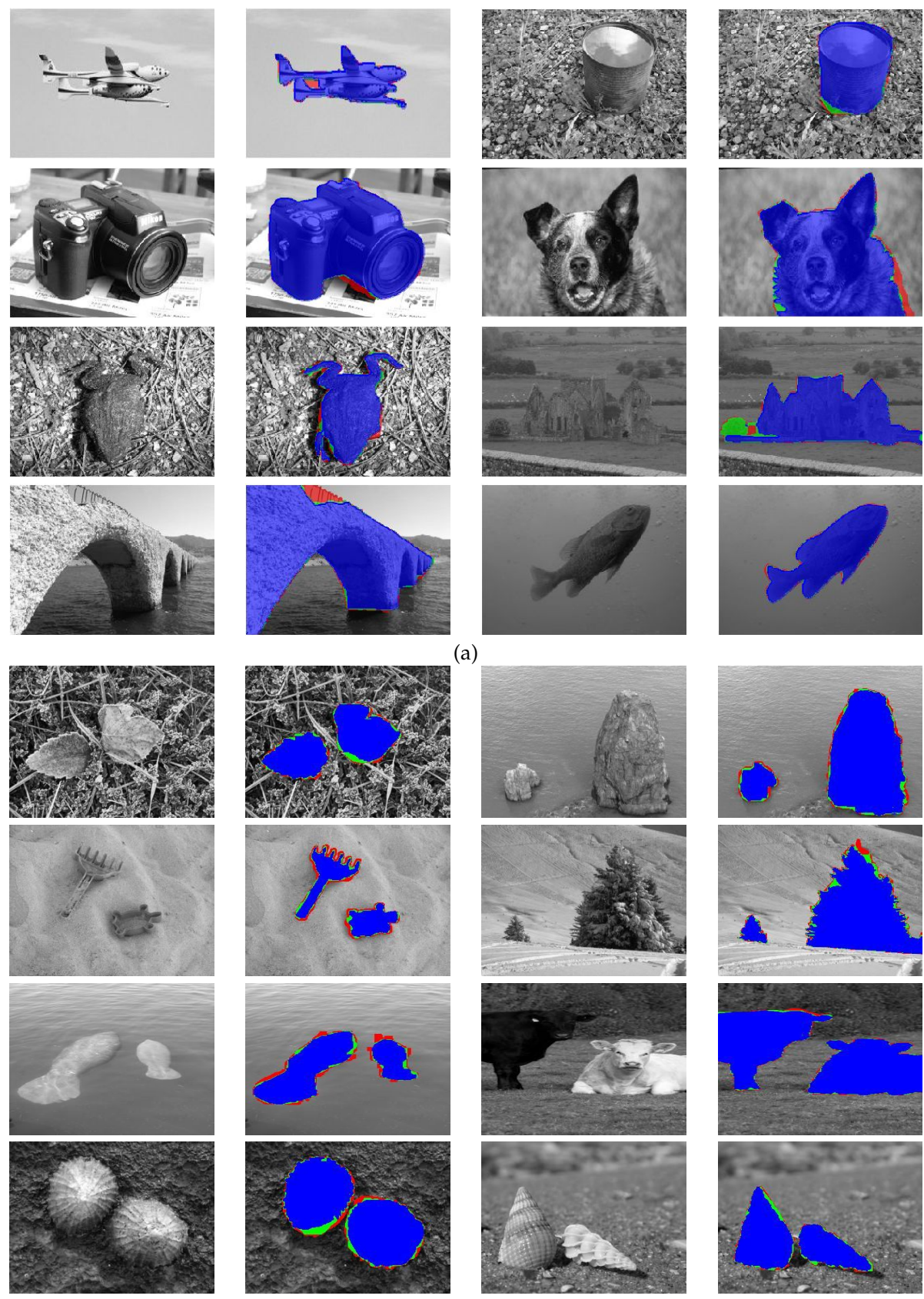

(b)

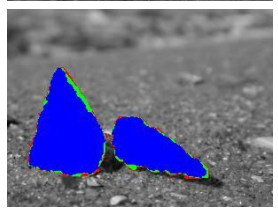

Fig. 4: A sample from the evaluation dataset. The images in (a) include one foreground object while those in (b) include two foreground objects. Each color represents a different number of votes given by the human subjects according to the following key: blue=3, green $=2$, and red $=1$. 

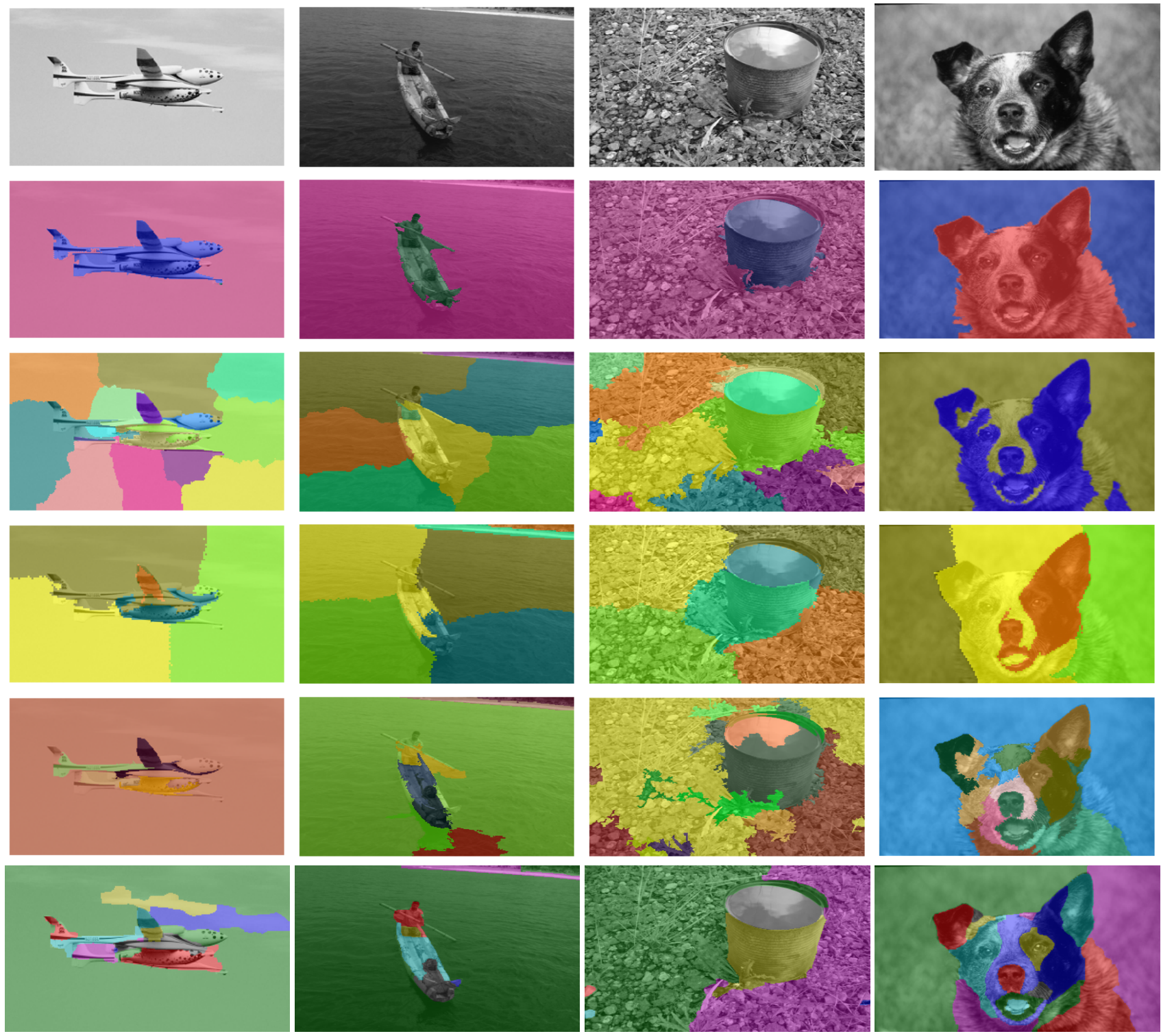

Fig. 5: A sample of the results obtained by applying our algorithm to images from the single object database compared to other algorithms. From top to bottom: original images, our method, SWA, Normalized cuts, Mean-shift, and Gpb. 

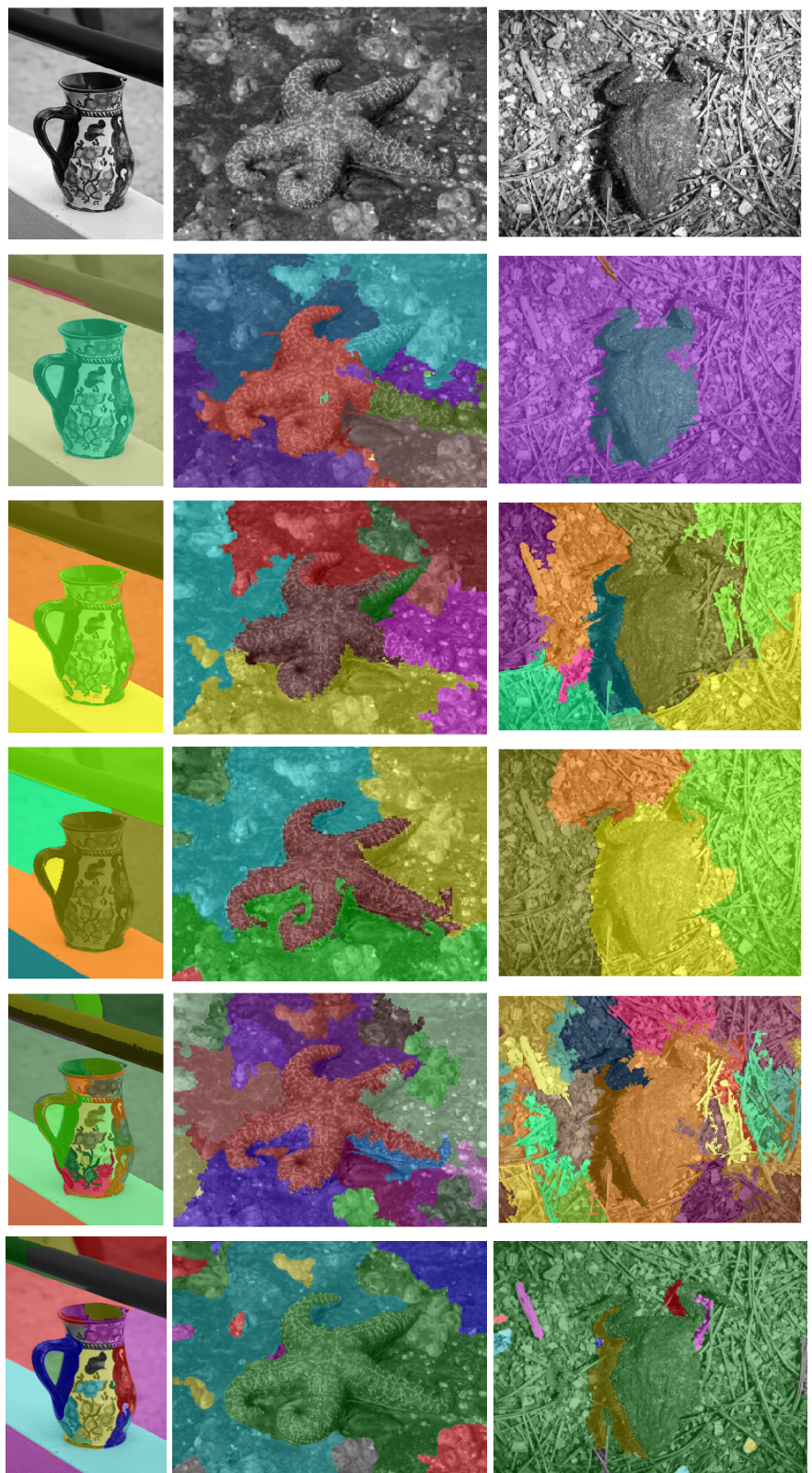

Fig. 6: A sample of the results obtained by applying our algorithm to images from the single object database compared to other algorithms. From top to bottom: original images, our method, SWA, Normalized cuts, Mean-shift, and Gpb. 

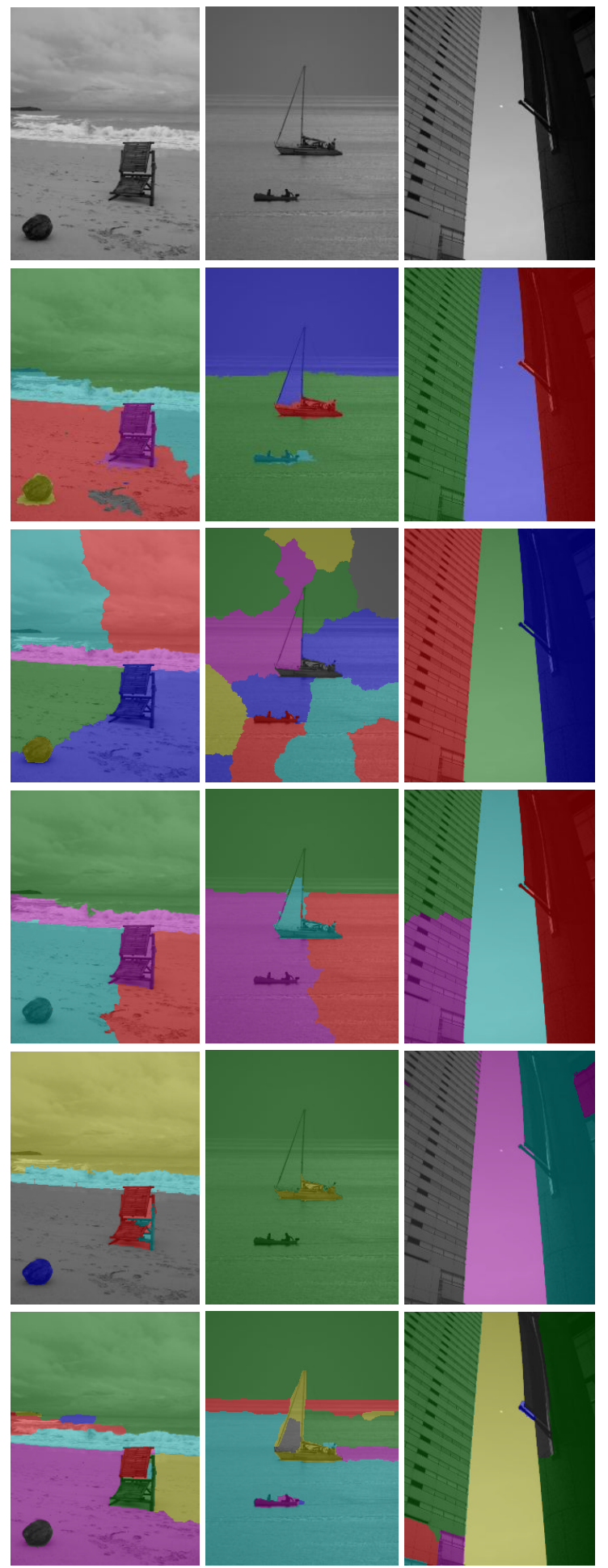

Fig. 7: A sample of the results obtained by applying our algorithm to images from the two objects database compared to other algorithms. From top to bottom: original images, our method, SWA, Normalized cuts, Mean-shift, and Gpb. 


\begin{tabular}{|c|c|}
\hline Algorithm & F-measure Score \\
\hline Our Method & $\mathbf{0 . 8 6} \pm 0.01$ \\
\hline SWA V1 & $0.83 \pm 0.02$ \\
\hline SWA V2 & $0.76 \pm 0.02$ \\
\hline N-Cuts & $0.72 \pm 0.02$ \\
\hline MeanShift & $0.57 \pm 0.02$ \\
\hline Gpb & $0.54 \pm 0.01$ \\
\hline
\end{tabular}

TABLE 1: Single segment coverage test results for the single object dataset.

\begin{tabular}{|c|c|c|c|}
\hline Algorithm & F-measure average & F-measure larger object & F-measure smaller object \\
\hline Gpb & $\mathbf{0 . 7 2} \pm 0.02$ & $0.70 \pm 0.02$ & $\mathbf{0 . 7 5} \pm 0.02$ \\
\hline Our Method & $0.68 \pm 0.05$ & $0.70 \pm 0.02$ & $0.65 \pm 0.03$ \\
\hline SWA V1 & $0.66 \pm 0.06$ & $\mathbf{0 . 7 4} \pm 0.03$ & $0.57 \pm 0.04$ \\
\hline SWA V2 & $0.61 \pm 0.07$ & $0.71 \pm 0.03$ & $0.50 \pm 0.04$ \\
\hline N-Cuts & $0.58 \pm 0.06$ & $0.66 \pm 0.04$ & $0.49 \pm 0.04$ \\
\hline MeanShift & $0.61 \pm 0.02$ & $0.65 \pm 0.03$ & $0.58 \pm 0.03$ \\
\hline
\end{tabular}

TABLE 2: Single segment coverage test results for the two objects dataset.

\begin{tabular}{|c|c|c|}
\hline Algorithm & $\begin{array}{c}\text { Averaged F-measure } \\
\text { Score }\end{array}$ & $\begin{array}{c}\text { Average number } \\
\text { of fragments }\end{array}$ \\
\hline Our Method & $0.87 \pm 0.02$ & $\mathbf{2 . 6 6} \pm 0.30$ \\
\hline SWA V1 & $0.89 \pm 0.01$ & $3.92 \pm 0.35$ \\
\hline SWA V2 & $0.86 \pm 0.01$ & $3.71 \pm 0.33$ \\
\hline N-Cuts & $0.84 \pm 0.01$ & $3.12 \pm 0.17$ \\
\hline Gpb & $0.88 \pm 0.02$ & $8.20 \pm 0.68$ \\
\hline MeanShift & $0.88 \pm 0.01$ & $12.08 \pm 0.96$ \\
\hline
\end{tabular}

TABLE 3: Fragmented coverage test results for the single object dataset.

two object dataset performed significantly worse on the single object dataset due to over-fragmentation. The next best score is achieved by the SWA algorithm utilizing its full set of features. Note that the performance of the mean shift algorithm suffers since this implementation does not handle texture.

In the second test, we permitted a few segments to cover the foreground by combining segments whose area overlaps considerably with the foreground object. Then for each union of fragments, we measured the $F$-measure score and the number of segments comprising it. The results of the fragmentation test for the both datasets are given in Tables 3-4.

In this test the averaged $F$-measure of the different algorithms is fairly similar. Yet, our method achieved considerably less fragmentation compared to the other methods, for both databases. A sample of results of applying our algorithm to images from the two databases is shown in Figures 5-7

In addition to this evaluation we have also evaluated our method on the Berkeley Image Segmentation Database [34]. Our algorithm (run with the same parameters as in the previous experiments) achieved an F-score of 0.52 on region covering test and 0.55 on the segmentation boundary test. These performances are comparable to the results achieved by other leading methods [32], [28] (region cover scores of 0.53-0.58 and boundary score of $0.59-0.66$ ), but inferior to those of [39] (respectively scores 0.65 and 0.74). A sample of results obtained for the Berkeley database [34] is shown in Figure 9. 


\begin{tabular}{|c|c|c|}
\hline Algorithm & $\begin{array}{c}\text { Averaged F-measure } \\
\text { Score }\end{array}$ & $\begin{array}{c}\text { Average number } \\
\text { of fragments }\end{array}$ \\
\hline Our Method & $0.85 \pm 0.03$ & $\mathbf{1 . 6 7} \pm 0.25$ \\
\hline SWA V2 & $0.85 \pm 0.03$ & $2.27 \pm 0.46$ \\
\hline N-Cuts & $0.84 \pm 0.04$ & $2.64 \pm 0.34$ \\
\hline Gpb & $0.84 \pm 0.01$ & $2.95 \pm 0.26$ \\
\hline SWA V1 & $0.88 \pm 0.04$ & $3.13 \pm 0.75$ \\
\hline MeanShift & $0.78 \pm 0.05$ & $3.65 \pm 0.75$ \\
\hline
\end{tabular}

\begin{tabular}{|c|c|c|c|c|}
\hline \multirow{2}{*}{ Algorithm } & \multicolumn{2}{|c|}{ Larger segment } & \multicolumn{2}{c|}{ Smaller segment } \\
& Average & Average & Average & Average \\
& F-measure & fragmentation & F-measure & fragmentation \\
\hline Our Method & $0.87 \pm 0.01$ & $\mathbf{2 . 0 0} \pm 0.16$ & $0.84 \pm 0.02$ & $\mathbf{1 . 3 3} \pm 0.09$ \\
\hline SWA V2 & $0.88 \pm 0.02$ & $2.76 \pm 0.30$ & $0.82 \pm 0.02$ & $1.77 \pm 0.16$ \\
\hline Gpb & $0.87 \pm 0.02$ & $3.60 \pm 0.30$ & $0.81 \pm 0.02$ & $2.30 \pm 0.26$ \\
\hline SWA V1 & $0.91 \pm 0.01$ & $3.88 \pm 0.46$ & $0.84 \pm 0.02$ & $2.37 \pm 0.29$ \\
\hline N-Cuts & $0.88 \pm 0.02$ & $3.34 \pm 0.20$ & $0.80 \pm 0.03$ & $1.93 \pm 0.14$ \\
\hline MeanShift & $0.85 \pm 0.02$ & $4.49 \pm 0.42$ & $0.71 \pm 0.03$ & $2.81 \pm 0.33$ \\
\hline
\end{tabular}

TABLE 4: Fragmented coverage test results for the two objects dataset. The tables show the average F-measure over both objects (top) and the results according to object size (bottom).

\section{SUMmARY}

We have presented an approach to image segmentation that is almost parameter-free. Our approach uses a bottom-up aggregation procedure in which regions are merged based on probabilistic considerations. The framework utilizes adaptive parametric distributions whose parameters are estimated locally using image information. Segmentation relies on an integration of intensity and texture cues, with priors determined by the geometry of the regions. The method is modular, and can readily be extended to handle additional cues. We further applied the method to a large database with manually segmented images and compared its performance to several recent algorithms.

\section{REFERENCES}

[1] A. K. Jain and R. C. Dubes, Algorithms for clustering data. Upper Saddle River, NJ, USA: Prentice-Hall, Inc., 1988.

[2] Y. Ma, H. Derksen, W. Hong, and J. Wright, "Segmentation of multivariate mixed data via lossy data coding and compression," IEEE Transactions on Pattern Analysis and Machine Intelligence, vol. 29, pp. 1546-1562, 2007.

[3] S.Geman and D.Geman, "Stochastic relaxation, gibbs distributions and the bayesian restoration of images," Pattern Analysis and Machine Intelligence, IEEE Transactions on, vol. 6, no. 6, pp. 721-741, 1984.

[4] B. S. Manjunath and R. Chellappa, "Unsupervised texture segmentation using markov random field models," IEEE Trans. Pattern Anal. Mach. Intell., vol. 13, no. 5, pp. 478-482, 1991.

[5] D. Mumford and J. Shah, "Optimal approximations by piecewise smooth functions and associated variational problems," Communications on Pure and Applied Mathematics, vol. 42, no. 5, pp. 577-685, 1989.

[6] D. Cremers, "A variational framework for image segmentation combining motion estimation and shape regularization," Computer Vision and Pattern Recognition, IEEE Computer Society Conference on, vol. 1, p. 53, 2003.

[7] J. A. Sethian, Level Set Methods and Fast Marching Methods: Evolving Interfaces in Computational Geometry, Fluid Mechanics, Computer Vision, and Materials Science. Cambridge University Press, 1998.

[8] O. Veksler, "Image segmentation by nested cuts." in CVPR, vol. 1, 2000, pp. 339-344.

[9] Z. Wu and R. Leahy, "An optimal graph theoretic approach to data clustering: Theory and its application to image segmentation," IEEE Transactions on Pattern Analysis and Machine Intelligence, vol. 15, no. 11, pp. 1101-1113, 1993. 

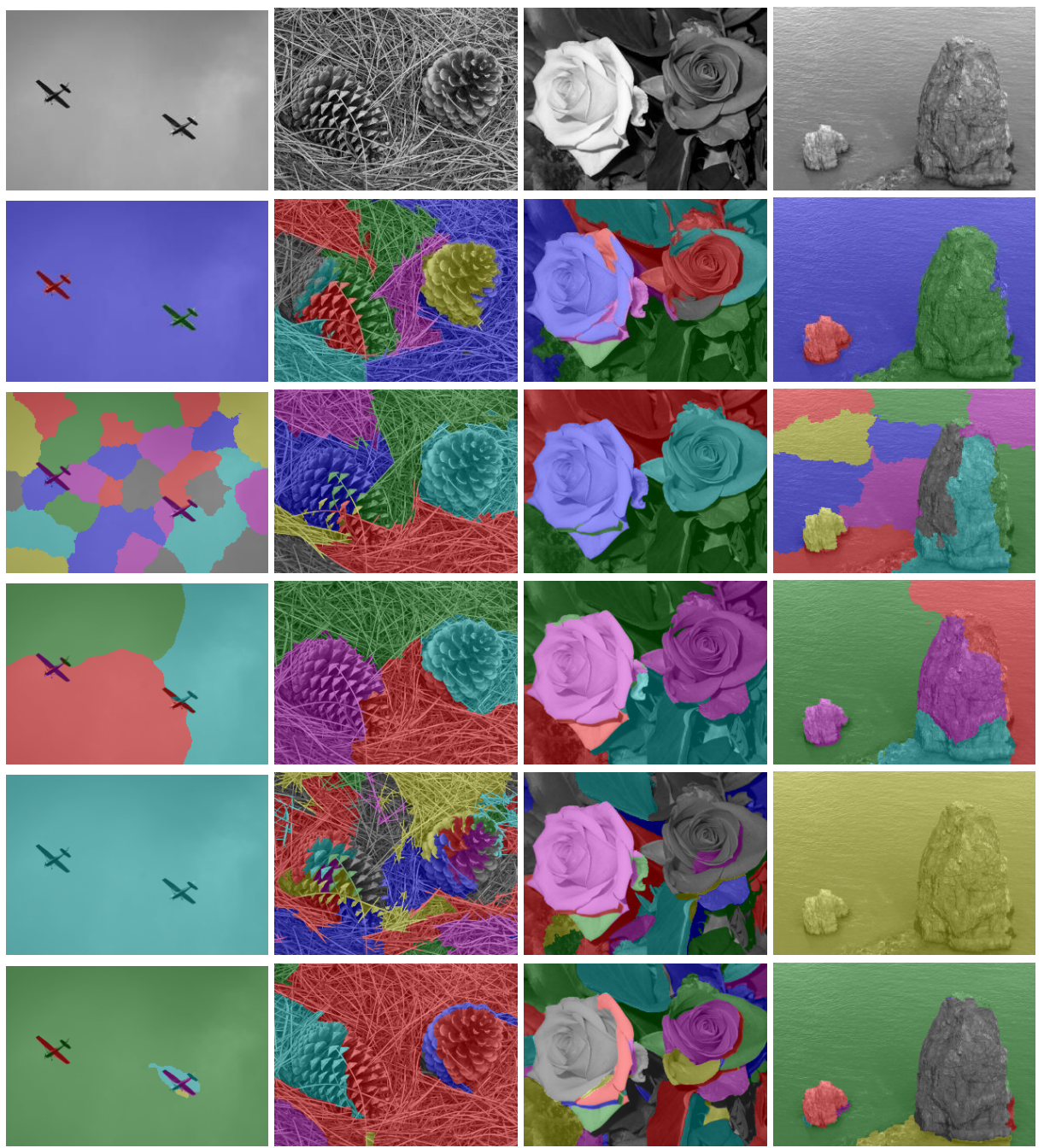

Fig. 8: A sample of the results obtained by applying our algorithm to images from the two objects database compared to other algorithms. From top to bottom: original images, our method, SWA, Normalized cuts, Mean-shift, and Gpb.

[10] I. J. Cox, S. B. Rao, and Y. Zhong, "Ratio regions: A technique for image segmentation." in International Conference on Pattern Recognition, 1996, pp. 557-564.

[11] S. Wang and J. M. Siskind, "Image segmentation with minimum mean cut." in ICCV, 2001, pp. 517-524.

[12] Y. Weiss, "Segmentation using eigenvectors: A unifying view," in ICCV '99: Proceedings of the International Conference on Computer Vision-Volume 2. Washington, DC, USA: IEEE Computer Society, 1999, p. 975.

[13] Y. Gdalyahu, D. Weinshall, and M. Werman, "Stochastic image segmentation by typical cuts." in CVPR, 1999, pp. 2596-2601.

[14] P. F. Felzenszwalb and D. P. Huttenlocher, "Efficient graph- based image segmentation," Int. J. Comput. Vision, vol. 59, no. 2, pp. 167-181, 2004.

[15] J. Shi and J. Malik, "Normalized cuts and image segmentation," TPAMI, vol. 22, no. 8, pp. 888-905, 2000.

[16] T. Cour, F. Bénézit, and J. Shi, "Spectral segmentation with multiscale graph decomposition." in CVPR (2), 2005, pp. 11241131.

[17] E. Sharon, M. Galun, D. Sharon, R. Basri, and A. Brandt, "Hierarchy and adaptivity in segmenting visual scenes," Nature, vol. 442, no. 7104, pp. 810-813, June 2006.

[18] X. Ren, C. C. Fowlkes, and J. Malik, "Cue integration in figure/ground labeling," in Advances in Neural Information 

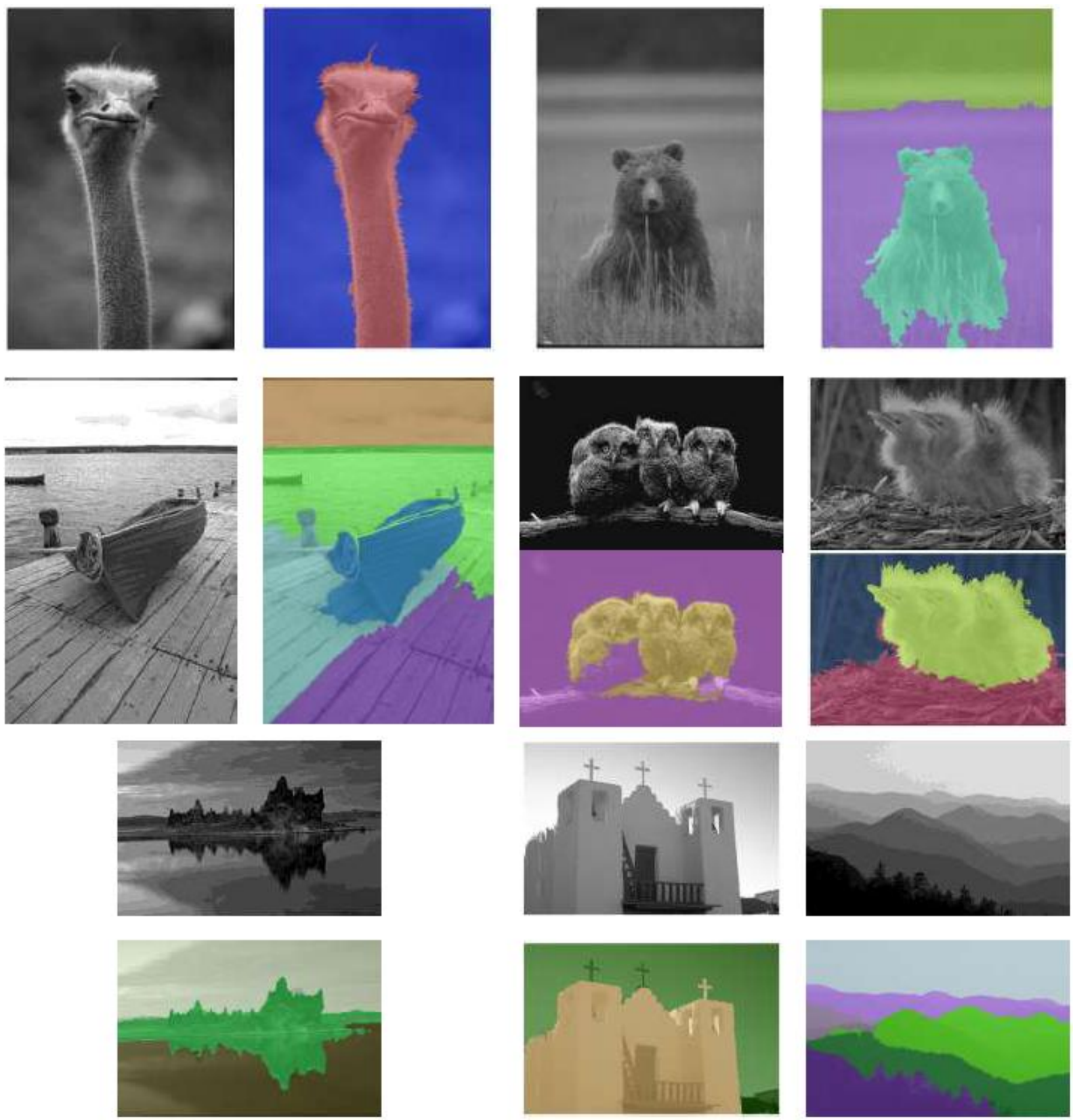

Fig. 9: A sample of the results obtained by applying our algorithm to images from the Berkeley database. 
Processing Systems 18, 2005.

[19] D. R. Martin, C. Fowlkes, and J. Malik, "Learning to detect natural image boundaries using local brightness, color, and texture cues." TPAMI, vol. 26, no. 5, pp. 530-549, 2004.

[20] A. Rabinovich, S. Belongie, T. Lange, and J. M. Buhmann, "Model order selection and cue combination for image segmentation." CVPR (1), pp. 1130-1137, 2006.

[21] R. O. Duda, P. E. Hart, and D. G. Stork, Pattern Classification (2nd Edition). Wiley-Interscience, November 2000.

[22] L. Vincent and P. Soille, "Watersheds in digital spaces: An efficient algorithm based on immersion simulations," TPAMI, vol. 13 , no. 6, pp. 583-598, 1991.

[23] H. T. Nguyen and Q. Ji, "Improved watershed segmentation using water diffusion and local shape priors." CVPR (1), pp. 985-992, 2006.

[24] T. Pavlidis and Y.-T. Liow, "Integrating region growing and edge detection," TPAMI, vol. 12, no. 3, pp. 225-233, 1990.

[25] D. K. Panjwani and G. Healey, "Markov random field models for unsupervised segmentation of textured color images," TPAMI, vol. 17, no. 10, pp. 939-954, 1995.

[26] S. Alpert, M. Galun, R. Basri, and A. Brandt, "Image segmentation by probabilistic bottom-up aggregation and cue integration." in Proceedings of the IEEE Conference on Computer Vision and Pattern Recognition, June 2007.

[27] C. Liu, W. T. Freeman, R. Szeliski, and S. B. Kang, "Noise estimation from a single image." CVPR (1), pp. 901-908, 2006.

[28] J. Malik, S. Belongie, T. K. Leung, and J. Shi, "Contour and texture analysis for image segmentation," International Journal of Computer Vision, vol. 43, no. 1, pp. 7-27, 2001.

[29] J. Malik and P. Perona, "Preattentive texture discrimination with early vision mechanisms," Journal of the Optical Society of America A, vol. 7, no. 5, 1990.

[30] P. O. Hoyer, "Non-negative matrix factorization with sparseness constraints," Journal of Machine Learning Research, vol. 5, pp. 1457-1469, 2004.

[31] P. Brodatz, "Textures: A Photographic Album for Artists and Designers". New York, NY ,USA: Dover Publications, 1966.

[32] M. Galun, E. Sharon, R. Basri, and A. Brandt, "Texture segmentation by multiscale aggregation of filter responses and shape elements." ICCV , pp. 716-723, 2003.

[33] A. Brandt, "Algebraic multigrid theory: The symmetric case," Applied Mathematics and Computation, vol. 19, no. 1-4, pp. 2356, 1986.

[34] D. Martin, C. Fowlkes, D. Tal, and J. Malik, "A database of human segmented natural images and its application to evaluating segmentation algorithms and measuring ecological statistics," ICCV (2), pp. 416-423, July 2001.

[35] F. Ge, S. Wang, and T. Liu, "Image-segmentation evaluation from the perspective of salient object extraction," in Proceedings of the 2006 IEEE Computer Society Conference on Computer Vision and Pattern Recognition - Volume 1. Washington, DC, USA: IEEE Computer Society, 2006, pp. 1146-1153. [Online]. Available: http:/ / portal.acm.org/citation.cfm?id=1153170.1153499

[36] "www.wisdom.weizmann.ac.il/ vision/databases.html."

[37] C. J. Van Rijsbergen, Information Retrieval, 2nd edition. Dept. of Computer Science, University of Glasgow, 1979.

[38] D. Comaniciu and P. Meer, "Mean shift: A robust approach toward feature space analysis," TPAMI, vol. 24, no. 5, pp. 603619, 2002.

[39] P. Arbelaez, M. Maire, C. Fowlkes, and J. Malik, "Contour detection and hierarchical image segmentation," EECS
Department, University of California, Berkeley, Tech. Rep. UCB/EECS-2010-17, Feb 2010. [Online]. Available: http:/ / www.eecs.berkeley.edu/Pubs/TechRpts/2010/EECS2010-17.html 